Recibido:

junio 15 de 2021

Aceptado:

octubre 20 de 2021

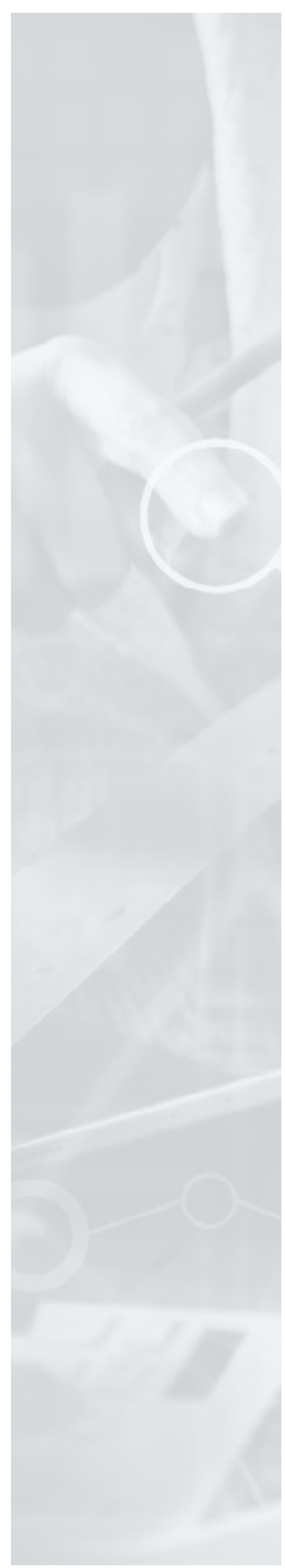

\section{Medición del control interno en la gestión de recursos humanos de la empresa Comercializadora Nacional, S.A.S}

\author{
Measurement of internal control in human resources \\ management of Comercializadora Nacional S.A.S
}

Astrid Elena Rivera Acevedo ${ }^{1}$

ariveraacev@uniminuto.edu.co.

https://orcid.org/0000-0001-9953-1271

David Alejandro Villacrés Moreno²

alejandrovillacres@hotmail.com

https://orcid.org/0000-0002-6238-9061

https://doi.org/10.22209/amr.v1n1a03

\section{Resumen}

La investigación que se muestra a continuación tuvo como objetivo general medir el nivel del control interno en la gestión de recursos humanos de la empresa Comercializadora Nacional, S.A.S. Para la realización de este trabajo, se tomaron en cuenta los aportes teóricos de Hernández (2014), Serrano (2017), Gamboa (2016), entre otros. Metodológicamente, se presenta un tipo de investigación mixta, con un diseño de campo y no experimental. Como técnica de recolección de datos se empleó la entrevista, para la cual se creó un guión de entrevista como instrumento; generando como resultado que siempre y cuando las empresas realicen sus actividades bajo la estructura de un sistema de control interno ajustado a sus necesidades, éste mediante la combinación de elementos de seguridad razonable, le proporciona la posibilidad de una mejor administración de los recursos disponibles, llegando a la conclusión que la implementación de controles en el proceso de nómina en la empresa en estudio, complementa funciones de gestión

1 Facultad de Contaduría Pública, Corporación Universitaria Minuto de Dios, Bello Antioquia. 2 Facultad de Ciencias Económicas y Administrativas, Universidad Cooperativa de Colombia. 
organizacional, evitando la materialización de riesgos representados en malos cálculos que pueden a su vez influir en la presentación de estados financieros fiables.

Palabras clave: beneficios actuariales; control interno; importancia relativa; pago de nómina.

\begin{abstract}
This research study aimed to measure the degree of internal control in the area of human resources in Comercializadora Nacional S.A.S. The theoretical contributions of Hernández (2019), Serrano (2017), Gamboa (2016), among others, were taken into account to carry out this study. A mixed methodology was used with a field and non-experimental design. The interview was used as the data collection technique, for which an interview script was developed. The results showed that the fact that companies carry out their activities according to the structure of an internal control system suited to the company needs, enables them to better manage the available resources through the combination of elements such as reasonable security. This study concludes that the company under study implements controls in the payroll process, which complement organizational management functions, thus mitigating risks of miscalculations that can in turn influence the presentation of reliable financial statements.
\end{abstract}

Keywords: actuarial benefits; internal control; relative importance; payroll payment.

\title{
Introducción
}

Actualmente, las empresas han reconocido la importancia de diseñar y aplicar medidas que posibiliten la protección integral de los recursos humanos, financieros y tecnológicos necesarios para la generación de información que refleje la situación económica real en los estados financieros; para lo cual se ha entendido el valor agregado que aporta el establecimiento de un adecuado sistema de control interno. El control interno es un modelo de organización; establece pautas de acción; linderos institucionales y aporta elementos para la mejora continua de los procesos y actividades operativas. El control interno constituye la base filosófica para lograr los objetivos corporativos; a través de él, existe la posibilidad de conocer los procesos y evaluar la gestión implementando actividades orientadas a optimizar las funciones de los trabajadores que hacen parte del talento humano en la unidad de negocios, y consecuentemente, impulsar la efectividad 
en cuanto a la implementación de estrategias de crecimiento y sostenibilidad en un ambiente dinámico como el actual.

En ese orden de ideas, Bonilla (2011) expone que el fundamento del control interno se forja inicialmente en el talento humano, por cuanto, a partir de allí, se direccionan los esfuerzos para desarrollar colaboradores ampliamente capacitados que constituyan talentos humanos que sumen esfuerzos en el alcance de la misión corporativa de la empresa. En otras palabras, la efectividad del control interno compuesto por políticas, normas y procedimientos, dependerá de la interpretación que cada colaborador le otorgue de acuerdo a las responsabilidades asumidas. Los colaboradores deben ser capaces que reconocer el qué, cómo y cuándo de las actividades implícitas en sus funciones, es decir; no basta que exista un control interno; es necesario que el talento humano sea capaz de ponerlo en práctica.

En este orden de ideas, esta investigación surge con el propósito de medir el nivel del control interno en el área de recursos humanos de la empresa Comercializadora Nacional, S.A.S, y con ello, valorar el tipo de acciones que emprenden los responsables del área de talento humano frente a la materialización del riesgo de control e implementación de medidas estratégicas para la representación fiel de la información financiera. Al respecto, según Auditool.org (2020) el riesgo de control constituye la consecuencia de una errónea representación de información que bien puede generar una importancia relativa a nivel individual, o ser el conjunto de otras; dicho error no ha sido detectado preventivamente o corregido de manera oportuna por los sistemas contables y de control.

Esta investigación se propone como una alternativa para validar la razonabilidad de la información financiera específicamente, en los componentes susceptibles de valoración económica en los procesos de recursos humanos. Para el desarrollo de este proyecto se aplicó una entrevista semiestructurada al personal de gestión humana, cuyo instrumento se enfocó en conocer los elementos claves para el cumplimiento de sus actividades y los aspectos relacionados al control interno.

\section{Materiales y métodos}

El presente estudio se caracteriza por ser una investigación descriptiva, no experimental y de campo. Según Hernández et al. (2014), los estudios descriptivos son aquellos que se encargan de analizar el objeto de estudio y presentar la información tal cual es observada, pudiendo ser interpretada y evaluada por el investigador. Del mismo modo, son trabajos no experimentales aquellos en los 
cuales los investigadores no tienen la intención de inferir o manipular la variable de estudio, sino por el contrario, mostrar la información tal fue recopilada y interpretada. A su vez, Corbetta (2007), expone que los estudios de campos son aquellos en los cuales, los investigadores tienen un acercamiento directo sobre las variables de estudio y la obtención de la información que posteriormente será objeto de análisis e interpretación.

Por su parte, en cuanto al instrumento de recolección de datos, se tomó como fundamento la definición de Arias (2012), el cual señala que este recurso es utilizado por el investigador con el fin de acercarse a la realidad del tema investigado y a su vez, poder adquirir información que se centre en la variable estudiada, indicando una línea investigativa veraz y concreta con fines posteriores de obtener respuesta a los objetivos planteados. Para el desarrollo de la presente investigación, el instrumento para la obtención de datos es definido mediante la entrevista; definida según Palella y Martins (2010), como una técnica que permite obtener la información a través de una o varias personas que ofrecen su opinión a través de una línea de interacción con el investigador, y que según se dé el desarrollo de la misma, se obtendrá la mayor información posible para el posterior análisis.

Siguiendo estos postulados, se establece que, a partir de la entrevista dirigida a la líder de talento humano de la empresa Comercializadora Nacional S.A.S, se pudo obtener información real sobre las acciones ejecutadas para el cumplimiento de los procesos de gestión humana, permitiendo ello conocer el nivel de aceptación y preparación frente al riesgo de control y sus consecuencias; además del aporte que hace la unidad estructural frente al control interno de la empresa.

Partiendo de la hipótesis que la administración del riesgo de control en el departamento de recursos humanos de la empresa Comercializadora S.A.S, se asume desde una posición reactiva, se procede de la siguiente manera: A través de la entrevista al jefe de recursos humanos, se solicita que describa detalladamente las actividades inherentes al cargo y sus directrices frente a los procesos relacionados a la administración del talento humano. La información obtenida fue analizada mediante una matriz de elaboración propia, tomando en cuenta los principios del modelo COSO; se asignaron una calificación cuantitativa que va de 0 a 5, con los siguientes criterios cualitativos frente al nivel de preparación del riesgo de control: básico, medio y óptimo.

El modelo COSO es una propuesta orientada a fortalecer el control interno en las organizaciones; fue definido para el año 1992; se basa en la necesidad imperiosa de que el nivel directivo y el resto del personal de una empresa, identifiquen la importancia y transcendencia del control interno como punto clave para integrar 
los procesos de organización y operación. Resulta importante destacar que en el año 2013 el modelo coso tuvo una actualización en el cual se demarcó a que el aspecto estratégico de la gestión organizacional, comprende la administración de riesgos como su pilar de ejecución (Hernández, 2019). Para el desarrollo de esta investigación, se tomó como referencia los cinco (5) componentes y diecisiete (17) principios del modelo COSO III, los cuales fueron elementos utilizados para medir a través del instrumento de recolección de datos aplicado, el sistema de control interno de la empresa en estudio.

\section{Resultados}

Una vez aplicado el instrumento de recolección de datos, a través del cual se obtuvo información valiosa sobre la situación real del objeto de estudio: control interno en la gestión del talento humano de la empresa Comercializadora Nacional, S.A.S, a continuación, se muestran los siguientes resultados:

En primer lugar, a través de la entrevista al jefe de recursos humanos, se obtuvo la información base para la investigación, en donde se describió detalladamente cada una de las actividades inherentes al cargo respectivo:

Tabla 1. Actividades del departamento de recursos humanos de la empresa Comercializadora Nacional, S.A.S.

\begin{tabular}{|l|l|}
\hline \multicolumn{1}{|c|}{ ACTIVIDADES } & \multicolumn{1}{|c|}{ DESCRIPCIÓN } \\
\hline $\begin{array}{l}\text { Ingreso de novedades } \\
\text { del personal y nómina }\end{array}$ & $\begin{array}{l}\text { Actividades orientadas a la administración de personal nuevo, ausentismos, terminación } \\
\text { de relación laboral, pago de recargos, deducciones. }\end{array}$ \\
\hline Generación de nómina & $\begin{array}{l}\text { La empresa cuenta con un software que, una vez ingresadas las novedades, se procede } \\
\text { con el procedimiento para la generación de la nómina. El resultado es revisado por la } \\
\text { contadora y la tesorera, donde las correcciones que surjan son realizadas por el jefe de } \\
\text { recursos humanos. }\end{array}$ \\
\hline Pago de nómina & $\begin{array}{l}\text { Se generan dos archivos planos. Uno para los pagos en efectivo de las personas que } \\
\text { aún no tienen cuenta bancaria que es entregado a la persona encargada de los pagos } \\
\text { a terceros. El otro archivo es el de las consignaciones que se realizan a través de la } \\
\text { plataforma del Banco Av villas. }\end{array}$ \\
\hline Elaboración de \\
comunicaciones & $\begin{array}{l}\text { Aviso del vencimiento de contratos: un mes antes de que se cumpla la fecha de } \\
\text { terminación de los contratos. } \\
\text { Referencia laboral. } \\
\text { Retiro de cesantías. } \\
\text { Solicitud de préstamo a Comfama. } \\
\text { Otras. }\end{array}$ \\
\hline
\end{tabular}




\begin{tabular}{|l|l|}
\hline \multicolumn{1}{|c|}{ ACTIVIDADES } & \multicolumn{1}{c|}{ DESCRIPCIÓN } \\
\hline $\begin{array}{l}\text { Gestión en Sistema } \\
\text { PILA }\end{array}$ & $\begin{array}{l}\text { Al terminar el mes se procede con la generación de la planilla integrada de liquidación de } \\
\text { aportes de acuerdo con los parámetros del sistema; luego se genera un archivo plano que } \\
\text { es enviado a la plataforma del operador ARUS y allí se genera la PILA con las novedades } \\
\text { de seguridad social para realizar la wrevisión y proceder con el pago. En esta actividad se } \\
\text { realiza un proceso de revisión y cálculos manuales para verificar que las bases tomadas } \\
\text { para el pago sean reales y coincidan con el valor del resumen de la nómina. }\end{array}$ \\
\hline $\begin{array}{l}\text { Interfaz de la seguridad } \\
\text { social al sistema } \\
\text { contable Aplinsa }\end{array}$ & $\begin{array}{l}\text { Este proceso es manual y se genera porque la información financiera y comercial son } \\
\text { administrados bajo otro software llamado Aplicaciones Integradas, Aplinsa SA. }\end{array}$ \\
\hline SGSST & $\begin{array}{l}\text { Al momento de la investigación, el jefe de recursos humanos se encontraba en } \\
\text { proceso de formación en administración en seguridad y salud en el trabajo, por lo } \\
\text { cual, aunado a la experiencia, se consolida la competencia para liderar dicho proceso. } \\
\text { Del mismo modo, se cuenta con el apoyo de la ARL Seguros Bolívar. }\end{array}$ \\
\hline
\end{tabular}

Fuente: elaboración propia, 2021.

Ahora bien; para dar respuesta al objetivo general de la investigación, en el cual se mide el nivel del control interno en la gestión de recursos humanos de la empresa en estudio; resulta importante señalar que la evaluación de la gestión del talento humano se realizó tomando como referencia los componentes y principios del modelo COSO III para la implementación del Sistema de Control Interno, que a su vez presenta como beneficios: disponer de herramientas integrales que permitan la actualización del sistema de control interno, apoyar a las organizaciones en cuanto a su administración para la puesta en marcha del sistema de control interno, y finalmente, contar con estándares para la ejecución y actualización de los principios que integran el modelo COSO III.

En ese sentido, en la tabla número 2 que a continuación se presenta, se muestra detalladamente los componentes y principios del modelo cosO III, los cuales constituyeron aspectos claves a medir en la gestión del talento humano en la empresa en estudio:

Tabla 2. Componentes y principios del modelo control interno cOSO III

\section{ESTRUCTURA DE LA ENTIDAD}

Entidad: Comercializadora Nacional S.A.S

División: Administrativa/Operativa

Unidad Operativa: Recursos Humanos

Función: Administración y cuidado del recurso humano de la empresa 


\begin{tabular}{|c|c|}
\hline COMPONENTES & PRINCIPIOS \\
\hline \multirow{5}{*}{$\begin{array}{l}\text { Ambiente de control/ } \\
\text { Gobierno y Cultura }\end{array}$} & 1. Ejercicio de supervisión de riesgos por medio del consejo de administración. \\
\hline & 2. Establecimiento de estructuras operativas. \\
\hline & 3. Definición de cultura deseada. \\
\hline & 4. Evidencia de compromiso con los valores clave. \\
\hline & 5. Retención del recurso humano capacitado y comprometido. \\
\hline \multirow{4}{*}{$\begin{array}{l}\text { Evaluación de riesgos/ } \\
\text { estrategia y objetivos }\end{array}$} & 6. Análisis del contexto empresarial. \\
\hline & 7. Apetito frente al riesgo. \\
\hline & 8. Evaluación de estrategias alternativas. \\
\hline & 9. Formulación de objetivos del negocio. \\
\hline \multirow{3}{*}{$\begin{array}{l}\text { Actividades de control/ } \\
\text { desempeño }\end{array}$} & 10. Identificación del riesgo. \\
\hline & 11. Evaluación sobre la gravedad del riesgo. \\
\hline & 12. Priorización y respuesta a los riesgos. \\
\hline \multirow{3}{*}{$\begin{array}{l}\text { Información y } \\
\text { comunicación }\end{array}$} & 13. Aprovechamiento de la información y la tecnología. \\
\hline & $\begin{array}{l}\text { 14. Comunicación de información sobre los objetivos y responsabilidades del control } \\
\text { interno. }\end{array}$ \\
\hline & 15. Información sobre el riesgo, la cultura y el desempeño. \\
\hline \multirow[t]{2}{*}{$\begin{array}{l}\text { Actividades de } \\
\text { monitoreo }\end{array}$} & $\begin{array}{l}\text { 16. Desarrollo de evaluación para comprobar el funcionamiento del sistema de } \\
\text { control interno y sus cambios significativos. }\end{array}$ \\
\hline & $\begin{array}{l}\text { 17. Comunicación sobre las deficiencias del control interno detectadas por medio de } \\
\text { las evaluaciones para mejorar la gestión del riesgo. }\end{array}$ \\
\hline
\end{tabular}

Fuente: elaboración propia, 2020

Del mismo modo, una vez aplicado el instrumento de recolección de datos a los sujetos informantes, en la tabla número 3, se muestran los resultados de la ubicación de las respuestas obtenidas a través de la entrevista realizada al jefe de personal de la empresa en estudio. Para tal efecto, según la realidad en la gestión de recursos humanos, a cada uno de los principios del modelo cosO III se le asignó un puntaje que osciló entre 0 a 5; teniendo presente que los aspectos no evidenciados o no respondidos por el sujeto informante, se califican como indeterminados, por cuanto no es válido asignar una calificación 0, considerando que las respuestas no fueron intervenidas, guiadas o profundizadas para tener un conocimiento más allá de lo que la persona entrevistada quiso expresar.

En consecuencia, conforme fue explicado en los párrafos anteriores, a continuación, se muestran los resultados de la valoración de los aspectos característicos del control interno según el Modelo Coso III. 
Tabla 3. Medición de la gestión de recursos humanos de la empresa Comercializadora Nacional S.A.S.

\section{Control Interno en el departamento de recursos humanos de la empresa "Comercializadora Nacional S.A.S"}

Evidencia frente al cumplimiento de los principios establecidos en la tabla 2. Cada uno de los aspectos descritos a continuación, corresponde la acción indicada por el sujeto informante frente a los principios del modelo COSO III.

La nómina a pagar al igual que otras actividades susceptibles de valoración económica están sujetas a un proceso de control previo ejercido por la contadora y la tesorera de la empresa.

Los procesos inherentes a la gestión de talento humano en su totalidad, se encuentran a cargo de una sola persona.

Existe total compromiso por parte de la persona encargada del proceso de gestión humana para dinamizar la cultura para una gestión oportuna de cada proceso del talento humano.

Indeterminado

Los responsables de la gestión del recurso humano se encuentran en proceso de formación en aspectos de seguridad y salud en el trabajo. Del mismo modo, existe la política de retener a los colaboradores capacitados y comprometidos.

Indeterminado

No hay segregación de funciones, por tanto, corresponde a una debilidad frente a la detección oportuna, valoración y gestión de riesgos en los macro procesos de gestión humana.

Indeterminado

Indeterminado

Se hace revisión antes de proceder con los pagos. Ello indica que existe una posibilidad de control previo antes de emitirse los pagos para detectar errores u omisiones dentro del proceso de gestión humana.

Indeterminado

Los procesos de revisión no están estandarizados, por tanto, dentro de la gestión del talento humano se dificulta la priorización y respuesta oportuna a los riesgos detectados.

Las revisiones del proceso de recurso humano susceptible de valoración económica se ejecutan de forma manual.

Indeterminado

Los errores de los procesos en la gestión de talento humano son detectados y corregidos a tiempo.

Indeterminado

Indeterminado

Fuente: elaboración propia, 2020

Ahora bien, luego de la actividad investigativa a través de la cual se recolectó información valiosa frente a la realidad que caracteriza la gestión de talento humano de la empresa en estudio, los resultados obtenidos muestran aspectos altamente positivos y otros por mejorar dentro de la ejecución de los procesos inherentes a la administración del recurso humano. Es así como en la figura siguiente se muestran las fortalezas dentro de proceso evaluado: 
Figura 1. Fortalezas en la gestión del talento humano de la Comercializadora Nacional, S.A.S

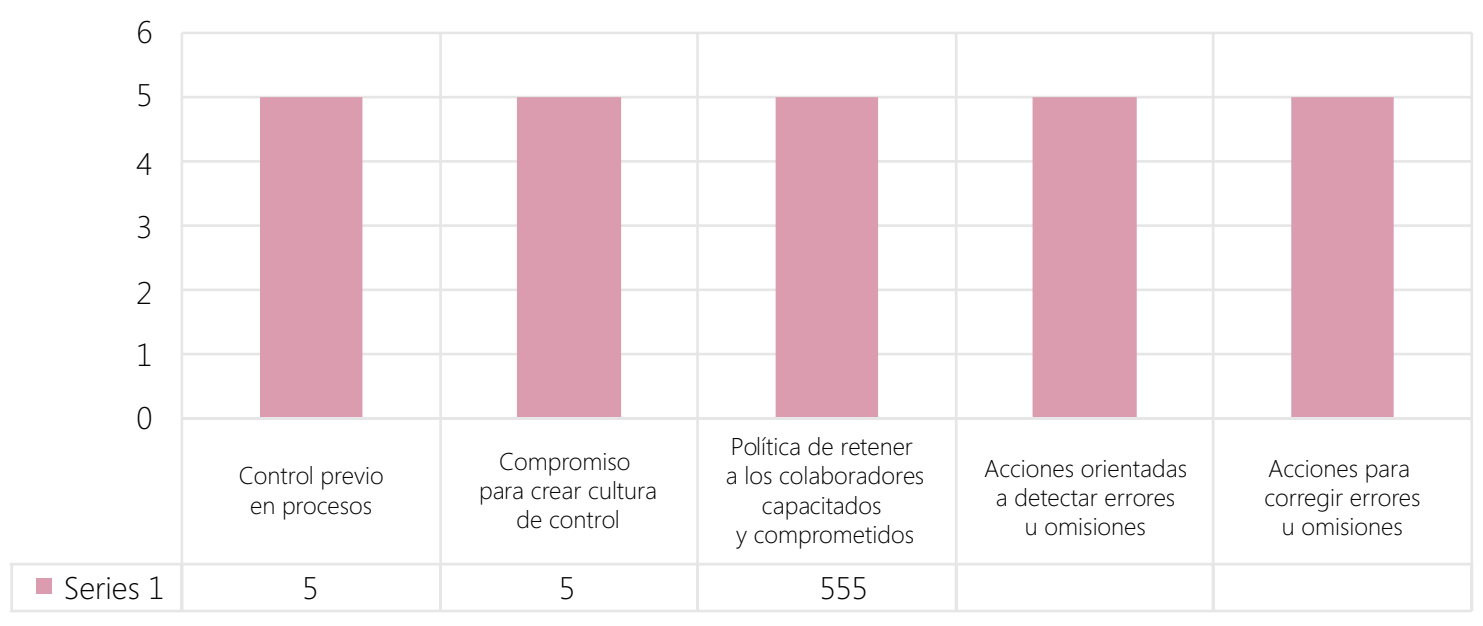

Fuente: Elaboración propia, 2021.

Como puede observarse en la figura anterior, dentro de los aspectos que constituyen fortalezas en la gestión de talento humano en la empresa Comercializadora Nacional, S.A.S, nos encontramos algunas directrices emanadas desde la gerencia orientadas a detectar y corregir errores en el cumplimiento del control interno y de las normas del ordenamiento jurídico colombiano en torno a actividades de recursos humanos, cada una de ellas con una ponderación de 5 . En ese sentido, aunado a lo anterior, mantener colaboradores con competencias para la ejecución de sus funciones y al mismo tiempo, crear en los mismos una cultura del control en el quehacer de las actividades, conlleva a posicionar a la unidad de recursos humanos en un elemento estratégico en la organización, por cuanto, la efectividad en la ejecución de las labores bajo su responsabilidad, contribuye significativa en la protección de los recursos financieros y posterior emisión de informes que muestren una situación razonablemente presentada.

Del mismo modo, paralelamente a los aspectos que constituyen fortalezas, dentro de la realidad que cobija la gestión de recursos humanos en la empresa, Comercializadora Nacional, S.A.S, se evidenciaron algunos aspectos que requieren ser atendidos y mejorados de cara a fortalecer el sistema de control interno existente: 
Figura 2. Debilidades en la gestión del talento humano de la Comercializadora Nacional, S.A.S

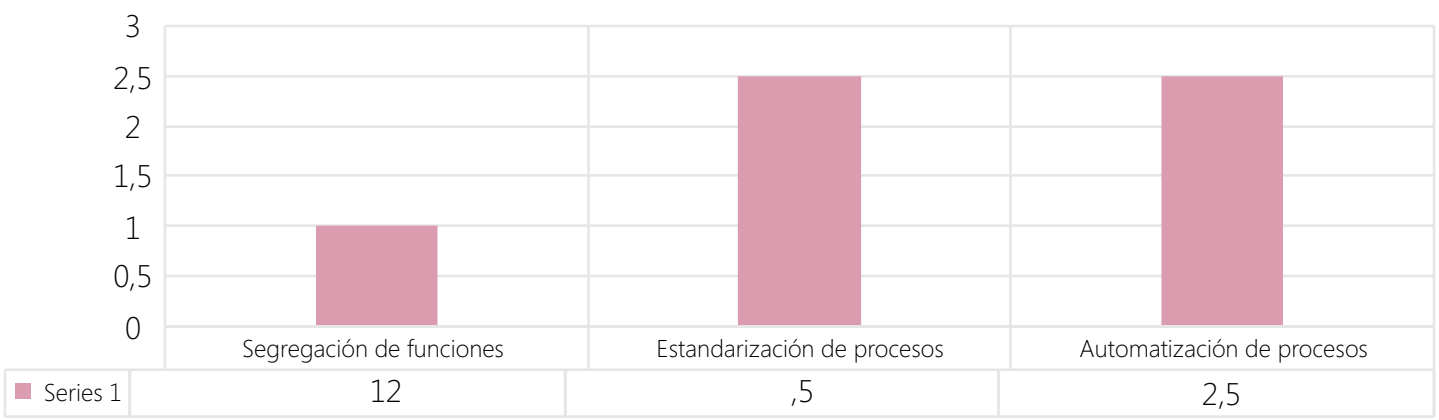

Fuente: Elaboración propia, 2021.

Si bien es cierto que la empresa en estudio, tiene dentro de sus fortalezas, acciones orientadas a fortalecer la cultura de control interno, también es cierto que los resultados de esta investigación demuestran algunas realidades que representan situaciones a mejorar. En ese sentido, conforme puede evidenciarse en la figura anterior, el hecho que la empresa no cuente con documentos internos que delimiten las funciones del personal, al igual que sus procesos inherentes a la administración de recursos humanos se realicen de forma manual y además, que tales procesos no estén estandarizados, ello dificulta en primera instancia, que el personal pueda comprender sus verdaderas responsabilidades y por ende, representa una debilidad que no contribuye a prevenir o reducir el riesgo de errores o irregularidades y en especial el fraude interno en la organizaciones.

Lo antes señalado representa una oportunidad de mejora frente a la necesidad de optimizar los procesos y establecer líneas de acción para que la empresa en estudio pueda consolidar una fuerza de trabajo interna que sea capaz de garantizar la implementación de un sistema de control interno sólido que coadyuve al alcance de sus objetivos institucionales.

\section{Discusiones}

Las reflexiones sobre los resultados obtenidos en este trabajo de investigación están orientadas en primer lugar, a esbozar algunos aspectos resaltantes en materia de control interno frente al desarrollo organizacional. Del mismo modo, se realiza una confrontación teórica frente a las fortalezas y debilidades que han resultado de la aplicación de los instrumentos de recolección de datos, con el firme propósito de aportas ideas claras que permitan consolidar el sistema de control interno de la empresa en estudio. 
En este sentido, se puede referir que el hecho de que las empresas realicen sus actividades bajo la estructura de un sistema de control interno apropiado según su estructura organizativa y recursos, les proporciona una mayor posibilidad de lograr la efectividad en la administración de los recursos disponibles, por cuanto el modelo de control en la gestión les permite contar con un sistema de información blindado con elementos seguridad razonable (Rivera 2015); gestión estratégica basada en resultados como lo manifiestan Garzón y Segovia (2011) y finalmente, contribuye a evitar lo que Herrera y Naranjo (2019) denominan como irregularidades en las operaciones.

A su vez, según lo señalado por Marín (2014), el control interno representa una herramienta administrativa de gran importancia en la organización, por cuanto, a través de él, puede ser posible la identificación y la valoración de los riesgos; al igual que el establecimiento de medidas de correctivas. En ese sentido, lo antes expuesto constituye una oportunidad para que en la ejecución de la gestión de recursos humanos pueda establecerse los parámetros necesarios para la detección de errores u omisiones que vayan en detrimento de la razonabilidad de la información financiera y el propio ordenamiento jurídico vigente. Por su parte, Valqui y Aguinaga (2016) hace especial énfasis en la responsabilidad que tiene la alta gerencia en la consolidación del sistema de control interno como una acción estratégica que debe permear en todos los procesos; lo que a su vez motiva a referir, que la unidad de recursos humanos es pieza clave dentro de este proceso de establecimiento de cultura de control.

Lo referido en el párrafo anterior coincide con lo señalado por Choix y Velázquez (2010), los cuales manifiestan que el control interno es una herramienta que se consolida como pieza clave para el desarrollo operativo y consecuentemente, financiero. En ese sentido, exponen los autores que un modelo de gestión bajo la estructura de control interno es propicio para todas las empresas independientemente de su tamaño, estructura y naturaleza de sus operaciones, y diseñado de tal manera, que permita proporcionar una razonable seguridad en lo referente a la efectividad de las operaciones, la confiabilidad de la información financiera, el acatamiento de las leyes y regulaciones aplicables.

A su vez, Laski (2006) comparte ideas con Choix y Velázquez (2010), al afirmar que el control interno es un componente esencial en el aprendizaje organizacional y es la gerencia de la empresa, quien tiene la alta responsabilidad de dinamizar las responsabilidades frente a su establecimiento y fiel cumplimiento. Para Laski (2006), el ejercicio oportuno de un sistema de control interno debe estar condicionado a una estructura metodológica, donde haya claridad de cada uno de los procesos medulares y de apoyo dentro de la organización; y al mismo tiempo, 
se identifique las áreas consolidadas y por mejorar de cada proceso con el firme propósito de crear valores orientados a la calidad de la gestión organizacional.

En segundo lugar, frente a las fortalezas detectadas durante la aplicación del instrumento de recolección de datos, resulta importante resaltar las acciones orientadas desde la gerencia hacia la implementación de controles previos en el cumplimiento de las actividades inherentes a la gestión de talento humano. Sobre este particular Bello Villar (2019), establece que el control previo incide significativamente en los procesos de recursos humanos tales como: documentación del gasto causado, cumplimiento de requisitos de existencia y calidad de los gastos, programación de pagos y ejecución de los mismos. Es decir, según lo señalado por el autor, el control previo es una herramienta fundamental para la razonabilidad de la información y el carácter fiel de los datos que se reportan en los estados financieros.

Por su parte, Misayauri y Neyra (2015) exponen que, al aplicar un ambiente de control como herramienta de mejora, ello influye significativamente en la ejecución de pagos. A su vez Martell (2021) plantea que crear un ambiente de control, realizar actividades previas de control y garantizar un sistema de información, contribuye significativa a la disminución de errores en la ejecución de procesos y, por ende, se evita en gran medida la incorrección material en los estados financieros.

En este mismo orden de ideas, Rivera (2015) expone que, frente al desarrollo de los procesos e implementación de controles para el logro de los objetivos organizacionales, la gestión del nivel ejecutivo y operativo debe garantizar información confiable y más rápida; por ello, las empresas deben aumentar su capacidad de organización de tal forma que le permita desarrollar destrezas de control gerencial para la disminución los riesgos y, exigir al personal directivo una capacitación apta para con sus responsabilidades.

Finalmente, respecto a las debilidades que resultaron de la aplicación del instrumento de recolección de datos, se puede especificar la falta de manuales de funciones que permita delimitar las resposabilidades de cada uno de los colaboradores, sobre lo cual Rocca y García (2016) plantean que el establecimiento de manuales administrativos favorece en la optimización de todos los recursos: tiempo, esfuerzos y cumplimiento de las instrucciones distinguiendo las responsabilidades de cada área o cargo y la orientación e información para todos los que interactúan dentro de la empresa. A su vez, Vivanco (2017) manifiesta que los manuales de procedimientos son la mejor herramienta, idónea para plasmar el proceso de actividades específicas dentro de una organización en las cuales 
se especifican políticas, aspectos legales, procedimientos, controles para realizar actividades de una manera eficaz y eficiente.

Por su parte, la no estandarización de procesos y la realización de los mismos de forma manual, igualmente representan situaciones que deben ser atendidas por la gerencia de la empresa. Al respecto, Moguillansky (2005) señala que la incorporación de la tecnología de la información a la empresa implica mejorar la eficiencia, productividad, eficiencia y rentabilidad de la unidad de negocios, para lo cual, se requiere de un entrenamiento, adaptación y en cierto sentido un cambio en la cultura laboral. Por su parte, Peña (2015) indica que la optimización de las unidades de proceso, determinan valor apropiado para las variables de operación proporcionen un rendimiento económico máximo para el proceso.

En consonancia a lo anterior, el departamento de recursos humanos tiene participación activa en el proceso de suplir lo que Paiva (2013) denomina una necesidad administrativa, por medio de unas medidas no documentadas, que están aportando al control interno de toda la organización, logrando de esta manera, según lo indica Gamboa (2016) la mejora de los procesos contables, administrativos y económicos para fortalecer el entorno de control y minimizar la materialización del riesgo de control que está directamente relacionado con la información errónea existente en las cuentas contables y que no es detectada de manera oportuna (Auditool.org, 2020).

En ese sentido, para la empresa en estudio, dentro de las actividades neurálgicas en la gestión del talento humano, habitualmente la información que se genera de los procesos de nómina son remitidos a través de una interfaz a los sistemas contables para alimentar rubros como obligaciones laborales, gastos de personal y otras cuentas relacionadas; completando así la información financiera que permite el cumplimento de obligaciones frente al Estado y demás entidades, cuya omisión genera sanciones a las empresas, de aquí la importancia que el proceso sea desarrollado con calidad y parámetros de control de acuerdo con el postulado de (Posso Robledo \& Barrios Barrios, 2014).

\section{Conclusiones}

En esta investigación se ha desarrollado una medición del nivel de control interno al área de recursos humanos de la empresa Comercializadora Nacional S.A.S. Para llegar a las consideraciones finales de este trabajo, se tomaron como referencia los componentes y principios del modelo de control coso. 
Los resultados demuestran que en efecto la empresa tiene altos estándares de cumplimiento de elementos de control interno orientados a garantizar que la información sea fiel en la gestión de recursos humanos; acorde a los requerimientos para la preparación de los estados financieros. Entre los aspectos a resaltar se encuentran: iniciativa para ejercer el control previo en las actividades operativas de talento humano, específicamente en todo lo relacionado a compromisos laborales y ejecución de pagos. Del mismo modo, el fomento de la cultura de control, la retención de colaboradores con amplias competencias en los procesos; así como las acciones orientadas a detectar y corregir errores u omisiones; son fortalezas de la entidad objeto de estudio y que deben potencializarse de cara a atender aquellas situaciones pendientes a mejorar.

En ese sentido, de acuerdo a los hallazgos obtenidos en esta investigación, se determinó que la empresa implementa medidas de control interno en el proceso de nómina, complementando así las funciones de gestión organizacional; evitando la materialización de riesgos representados en malos cálculos y pagos que disminuyen las utilidades del periodo y que pueden consecuentemente afectar la generación de estados financieros fiables. Las actividades ejecutadas en el área de recursos humanos están relacionadas con beneficios a los empleados, la retención en salarios con aplicación del procedimiento 1 o 2 contemplados en los artículos 385 y 386 del Estatuto Tributario vigente, entre otras.

Aunado a lo anterior, la empresa en estudio tiene el reto de crear los manuales descriptivos de cargos que le permita a los colaboradores tener claridad del tipo, periodicidad y alcance de cada una de las funciones y/o tareas a ejecutar. Por consiguiente; la estandarización y automatización de procesos también corresponde a situaciones que deben ser fortalecidas por cuanto el mejoramiento de todos los aspectos antes señalados, contribuirán a la seguridad razonable para la generación de información fiel en los estados financieros. Con la sistematización o estandarización de las actividades se realiza una gestión eficiente de los sistemas de control y se generan avances efectivos que se reflejan en todos los departamentos de la empresa; al respecto Acosta (2005), define la sistematización como la organización de la información que ya existe, con el fin de explicar los cambios sucedidos durante un periodo de tiempo o un proyecto aplicado, cuales son los factores que intervinieron, resultados y lecciones aprendidas.

Finalmente, se recomienda a la gerencia fijar la mirada hacia el plan estratégico de la organización, específicamente en su componente de control como una herramienta de mejoramiento continuo orientada a aumentar los niveles de cumplimiento frente a las políticas, normas y procedimientos que faciliten la ejecución de actividades en la administración del recurso humano. 


\section{Referencias}

Arias, F (2012). El Proyecto de Investigación. Editorial Episteme.

Auditool.org. (2020). ¿Qué es el riesgo de control en una auditoría de información financiera? https://www.auditool.org/blog/auditoria-externa

Bacallao, M. (2009). Génesis del control interno. Contribuciones a la economía.

Bello Villar, G. H. (2019). Incidencia del control previo en la ejecución de pagos de la sub gerencia de tesorería de la municipalidad provincial de Huarochirí 2018.

Bonilla, M. (2011). El Control Interno, camino para obtener la excelencia empresarial. https://www.auditool.org/blog/control-interno/961-el-control-interno-caminopara-obtener-la-excelencia-empresarial

Corbetta, P (2007). Metodología y Técnicas de Investigación Social. Editorial Mc Graw Hill, Madrid España.

Choix, M. R. A., \& Velazquez, L. C. E. A. (2012). La importancia del control interno en las pequeñas y medianas empresas en México Importance of internal control in small and medium enterprises in Mexico. Revista El Buzón de Pacioli, 12(76).

Gamboa, H. (2016). Caracterización del control interno de la gestión administrativa de las empresas comerciales del Perú: Caso Minera Andina FOR SAC.

Garzón Salazar, F. y Segovia Villavicencio, J. (2011). Diseño e implementación de un sistema de Control Interno para la Compañía "DATUGOURMET CIA. LTDA. Quito: Universidad Central de Ecuador.

González, J. (2013). Estudio sobre la competitividad de Pymes incubadas en Empreser de México. (Tesis de Maestría), Universidad Autónoma de Baja California, México.

Hernández, R, Fernández, C y Baptista, P. (2014). Metodología de la Investigación. Editorial McGraw-Hill.

Hernández, C. (2019). A propósito de la evolución del modelo de gestión de riesgos empresariales - COSO ERM. https://www.incp.org.co/ proposito-la-evolucion-del-modelo-gestion-riesgos-empresariales-coso-erm

Herrera, F. y Naranjo, A. (2019). Evaluación al control interno del componente bancos y nómina trabajadores para toma de decisiones de la Compañía La Tablita S.A. Machala.

Laski, J. P. (2006). El control interno como estrategia de aprendizaje organizacional: el modelo COSO y sus alcances en América Latina. Revista Gestión y estrategia, (30), 9-24.

Marín, M. S. C. (2014). El control interno basado en el modelo COSO. Revista de Investigación Valor Contable, 1(1).

Martell Javier, F. W. (2021). Control previo y la ejecución de pagos de tesorería en la DIRIS-Lima Este, 2021.

Misayauri Barbaran, I. A., \& Neyra Sandoval, E. B. (2015). Control previo en la ejecución de pagos en el área de tesorería de los colegios profesionales de la región Junín.

Moguillansky, G. (2005). La importancia de la tecnología de la información y la comunicación para las industrias de recursos naturales. CEPAL. 
Paiva, A. (2013). Control interno y su incidencia en la rentabilidad de empresa cobros del norte S.A. FAREM-Estelí. Medio ambiente, tecnología y desarrollo humano.Palella, S y Martins, F (2012). Metodología de la Investigación Cuantitativa. Editorial Mc Graw Hill Interamericana, S.A. Bogotá, Colombia.

Palella Stracuzzi, S., \& Martins Pestana, F. (2010). Metodología de la investigación cuantitativa. Caracas, Venezuela: FEDUPEL, Fondo Editorial de la Universidad Pedagógica Experimental Libertador.

Peña, A. M. A. (2015). La importancia de la optimización en la industria. Revista Virtualpro, Optimización de Procesos, Segunda Entrega, Tech. Rep, 159.

Posso, R. y Barrios, B. (2014). Diseño de un modelo de control interno en la empresa prestadora de servicios hoteleros eco turísticos nativos activos eco hotel la Cocotera, que permitirá el mejoramiento de la información financiera. repositorio. unicartagena.edu.co/handle/11227/2130

Rivera, F. (2015). Importancia del control interno en los negocios. Revista vinculando.

Roca, A. F., \& García, C. M. Q. (2016). Importancia de los manuales de procedimientos para la mejora de los procesos operativos en las agencias de aduanas. Revista Caribeña de Ciencias Sociales, (2016_10).

Serrano, C. (2017). El control interno como herramienta indispensable para una gestión financiera y contable eficiente en las empresas bananeras de Cantón Machala.

Valqui, G. L., \& Aguinaga, J. J. T. (2016). El sistema de control Interno: Una herramienta para el perfeccionamiento de la gestión empresarial en el sector construcción. Revista de Investigación de Contabilidad Accounting power for business, 1(1).

Vivanco Vergara, M. E. (2017). Los manuales de procedimientos como herramientas de control interno de una organización. Revista Universidad y Sociedad, 9(3), 247-252. 